\title{
Imaging of CAR T-Cells in Cancer Patients: Paving the Way to Treatment Monitoring and Outcome Prediction
}

\author{
Simone Krebs ${ }^{1}$, Vladimir Ponomarev ${ }^{2}$, Susan Slovin ${ }^{3}$, and Heiko Schöder ${ }^{1}$ \\ ${ }^{I}$ Molecular Imaging and Therapy Service, Department of Radiology, Memorial Sloan Kettering Cancer Center, New York, New York; \\ ${ }^{2}$ Radiochemistry and Molecular Imaging Sciences Service, Department of Radiology, Memorial Sloan Kettering Cancer Center, New \\ York, New York; and ${ }^{3}$ Guam Oncology Service, Department of Medicine, Memorial Sloan Kettering Cancer Center, New York, New \\ York
}

$\mathbf{I}_{n}$ 2013, the journal Science named cancer immunotherapy as the "breakthrough of the year" based on targeted approaches using chimeric antigen receptor (CAR) T-cells. CD19-specific CAR T-cell therapy has revolutionized the treatment landscape for patients with relapsed B-cell acute lymphocytic leukemia $(1,2)$. Similar successes have not been seen in patients with solid tumors, in part because of inconsistent expression of specific tumor antigens and physical impediments to T-cell trafficking, for example, for passing the blood-brain barrier or reaching metastatic disease in bone marrow. Other factors interfering with prolonged response to CAR T-cell therapy include loss of target antigen and T-cell exhaustion. These resistance mechanisms may be alleviated by CARs targeting 2 or more tumor antigens (NCT03019055), more sophisticated T-cell engineering techniques, or combination therapies of CARs with various immune checkpoint inhibitors. So far, it has remained unclear why certain cell therapies succeed and even provide durable clinical responses $(1,2)$ whereas others fail $(3)$.

Ideal monitoring of CAR T-cell therapies should include the ability to track T-cell migration, engagement with the antigenbearing tumor cells, as well as T-cell expansion and persistence at the tumor site-all essential steps for therapeutic efficacy. Imaging studies might perhaps also enable timely intervention to avoid potentially lethal systemic toxicity. Current clinical methods to monitor the infused cells include serum profiling of cytokines associated with T-cell activation, direct enumeration of tumorspecific T-cells in peripheral blood, and (repeated) tumor biopsies. Overall, the in vivo activation dynamics of engineered immune cells remain incompletely understood, as no means of real-time monitoring of the intratumoral milieu currently exists.

\section{PRINCIPLES OF CELL TRACKING}

Cell tracking by imaging requires the direct or indirect labeling of cells. Direct labeling is relatively simple and has been used clinically for several decades, for instance, with ${ }^{99 \mathrm{~m} T c}$ or ${ }^{111} \mathrm{In}-$ labeled leukocytes. More recently, ${ }^{89} \mathrm{Zr}$-oxine has been used for cell labeling and PET imaging (4). As a major disadvantage, the

For correspondence or reprints contact: Heiko Schöder, Department of Radiology, Molecular Imaging and Therapy Service, Memorial Sloan Kettering Cancer Center, 1275 York Ave., New York, NY 10065.

E-mail: schoderh@mskcc.org

Published online May 3, 2019.

COPYRIGHT (C) 2019 by the Society of Nuclear Medicine and Molecular Imaging. DOI: 10.2967/jnumed.119.227561 label is diluted over time when cells divide, resulting in decreased amounts of label per individual cell, narrowing the time window for cell tracking. In addition, direct cell labeling does not permit visualization of cell proliferation, cell activation, or cell death. So far, no clinical CAR T-cell studies using direct cell labeling have been reported.

Indirect labeling uses a reporter gene that is introduced into the genome of the cell and then translated into a protein (e.g., enzyme or cell surface receptor), engaging a radiolabeled probe. Stable expression of a reporter gene theoretically enables serial imaging of labeled cells over a prolonged time. As the reporter gene is passed on to cell progeny, imaging of expanding cell populations is possible, providing information about cell viability. One prerequisite is that the physical half-life of the isotope label matches the pharmacokinetics of the transplanted T-cells. Nevertheless, under clinical conditions, reporter gene imaging remains challenging and success stories are rare $(5,6)$. This is partly related to constraints in vector design and vector delivery, as well as the biologic and potentially immunogenic effects of reporter gene products on the engineered cells.

\section{CLINICAL IMAGING}

Herpes simplex virus type 1 thymidine kinase has been the most widely used reporter gene and is currently used to track CAR T-cells targeting interleukin-13 receptor $\alpha 2$ and prostatespecific membrane antigen in clinical studies (NCT00730613, NCT01082926, NCT01140373). Yaghoubi et al. reported the noninvasive detection of cytotoxic $\mathrm{T}$ lymphocytes in a patient with recurrent glioblastoma; cytotoxic $\mathrm{T}$ lymphocytes were engineered to express herpes simplex virus type 1 thymidine kinase and an interleukin-13 CAR, enabling PET imaging with the reporter probe ${ }^{18} \mathrm{~F}-\mathrm{FHBG}(5)$. The patient underwent gross tumor resection followed by serial autologous CD8 + CAR T-cell infusions (cumulative T-cell dose of $1 \times 10^{9}$ ) into the postsurgical cavity. Three days after the last T-cell dose, ${ }^{18} \mathrm{~F}$-FHBG was given intravenously, followed by PET imaging. PET/MR fusion images showed tracer accumulation at the postsurgical site, and also in the contralateral hemisphere, at a nonresected tumor site. Recently, these authors reported a small clinical trial in 7 patients with recurrent glioma, using the same approach. Although the imaging signal was relatively low (with SUVs below 1.0), this did not limit T-cell detection, because there was negligible background activity in normal brain. However, such low background activity cannot be expected in other body regions. Nonspecific radiotracer uptake 
was also noted in 1 patient (positive baseline scan before cytotoxic T-lymphocyte administration) (6).

Although this first clinical study is pivotal for the field, it highlights the current limitations in CAR T-cell imaging: detection of a small number of labeled cells in a large volume is confounded by the combination of low imaging signal from labeled cells and nonspecific background activity. This will be even more challenging when CAR T-cells are administered systemically rather than via intracavitary methods. The lack of a corresponding positive imaging signal could be limited by lack of sophisticated imaging modalities, suboptimal tumor-specific binding of T-cells and receptor engagement, or absence of T-cell activation, migration, and expansion. Studies can also be compromised by the immunogenicity of a xenogeneic (nonhuman) reporter gene (7).

\section{IMAGING MODELS}

Potential immunogenicity of the reporter gene product may be avoided when using human reporter gene systems (e.g., the human sodium iodide symporter, human norepinephrine transporter, human somatostatin receptor subtype 2 , human deoxycytidine, and thymidine kinase mutants (8), and prostate-specific membrane antigen) or using artificial constructs, such as a membrane-bound cell-surface antibody fragment binding small metal chelates (9). For instance, the human sodium iodide symporter reporter gene has been used for CAR T-cell tracking in prostate cancer mouse models (10). However, sodium iodide symporter is naturally found in stomach, salivary glands, lactating breast, and thyroid tissue, limiting the specificity of this approach (11). Expression of human somatostatin receptor subtype 2 has been evaluated in adoptively transferred CAR T-cells targeting intercellular adhesion molecule 1, which is overexpressed in anaplastic thyroid cancer, and imaged with ${ }^{68} \mathrm{Ga}$-DOTATOC PET/ CT (12). However, human somatostatin receptor subtype 2 internalizes on binding of the ligand, potentially interfering with T-cell function, and is physiologically expressed on immune cells, again limiting the specificity of this approach (13). Human deoxycytidine kinase double mutant has been tested in prostate-specific membrane antigen-targeted human CAR T-cells as a pyrimidine-specific PET reporter/suicide gene for imaging with ${ }^{18} \mathrm{~F}-\mathrm{FEAU}$ (14).

Recently, our group has tested a membrane-bound cell-surface antibody fragment using small metal chelates (e.g., DOTA) as a reporter probe, which is a purely synthetic molecule with no natural analogs. Modification by naturally occurring enzymes or competition for target binding by endogenous molecules is thereby avoided. Moreover, only cells modified with this construct should bind the radio-hapten, thus enabling exquisite specificity (9).

\section{NOVEL CONSTRUCTS}

CAR T-cells are currently generated using randomly integrating vectors, including $\gamma$-retroviral vectors, lentiviral vectors, and DNA transposons, to insert the CAR complementary DNA in the T-cell genome (15). Although effective, this approach may result in variegated gene expression, clonal expansion, oncogenic transformation, and transcriptional silencing (16-18). The emergence of targeted nuclease clustered regularly interspaced short palindromic repeats (CRISPR) provides a new means to specifically disrupt endogenous genes or target transgene delivery to chosen locations (19-21), potentially resulting in greater gene expression (22). A higher density of expressed reporter molecules should facilitate detection of the modified cells in vivo. "Armored"
CAR T-cells that also affect the tumor immune microenvironment may lead to greater efficacy of treatments (23).

\section{REAL-TIME APPLICATION}

Other T-cell imaging strategies take advantage of specific T-cell surface markers, such as CD8 (NCT03107663) (24). Another strategy is based on imaging T-cell activation with $2^{\prime}$-deoxy$2{ }^{\prime}{ }^{18}$ F-fluoro- $9-\beta$-D-arabinofuranosylguanine, a radiolabeled ana$\log$ of the DNA nucleoside guanine (NCT03142204) (25). The utility of these methods for CAR T-cell tracking has yet to be evaluated. Finally, serial imaging studies with probes binding to T-cell-inhibiting and -activating molecules, such as CTLA-4, PD-1/PD-L1, LAG-3, IL-2R, and OX40 (26-28), may enable understanding of interactions between CAR T-cells and the tumor microenvironment and, potentially, provide real-time response assessment.

\section{CONCLUSION}

Noninvasive imaging is a tool that remains to be exploited for assessing the systemic kinetics and functions of CAR T-cells in humans. The field is ripe with novel approaches currently in development.

\section{DISCLOSURE}

No potential conflict of interest relevant to this article was reported.

\section{REFERENCES}

1. Porter DL, Levine BL, Kalos M, Bagg A, June CH. Chimeric antigen receptormodified T cells in chronic lymphoid leukemia. N Engl J Med. 2011;365:725-733.

2. Sadelain M. CAR therapy: the CD19 paradigm. J Clin Invest. 2015;125:33923400 .

3. Morgan RA, Yang JC, Kitano M, Dudley ME, Laurencot CM, Rosenberg SA. Case report of a serious adverse event following the administration of $\mathrm{T}$ cells transduced with a chimeric antigen receptor recognizing ERBB2. Mol Ther. 2010;18:843-851.

4. Sato N, Wu H, Asiedu KO, Szajek LP, Griffiths GL, Choyke PL. ${ }^{89} \mathrm{Zr}$-oxine complex PET cell imaging in monitoring cell-based therapies. Radiology. 2015;275: 490-500.

5. Yaghoubi SS, Jensen MC, Satyamurthy N, et al. Noninvasive detection of therapeutic cytolytic T cells with ${ }^{18} \mathrm{~F}$-FHBG PET in a patient with glioma. Nat Clin Pract Oncol. 2009;6:53-58.

6. Keu KV, Witney TH, Yaghoubi S, et al. Reporter gene imaging of targeted T cell immunotherapy in recurrent glioma. Sci Transl Med. 2017;9:eaag2196.

7. Riddell SR, Elliott M, Lewinsohn DA, et al. T-cell mediated rejection of genemodified HIV-specific cytotoxic T lymphocytes in HIV-infected patients. Nat Med. 1996;2:216-223.

8. Lee JT, Moroz MA, Ponomarev V. Imaging T cell dynamics and function using PET and human nuclear reporter genes. Methods Mol Biol. 2018;1790:165180.

9. Krebs S, Ahad A, Carter LM, et al. Antibody with infinite affinity for in vivo tracking of genetically engineered lymphocytes. J Nucl Med. 2018;59:1894-1900.

10. Emami-Shahri N, Foster J, Kashani R, et al. Clinically compliant spatial and temporal imaging of chimeric antigen receptor T-cells. Nat Commun. 2018;9:1081.

11. Wapnir IL, van de Rijn M, Nowels K, et al. Immunohistochemical profile of the sodium/iodide symporter in thyroid, breast, and other carcinomas using high density tissue microarrays and conventional sections. J Clin Endocrinol Metab. 2003;88:1880-1888.

12. Vedvyas Y, Shevlin E, Zaman M, et al. Longitudinal PET imaging demonstrates biphasic CAR T cell responses in survivors. JCI Insight. 2016;1:e90064.

13. Elliott DE, Li J, Blum AM, Metwali A, Patel YC, Weinstock JV. SSTR2A is the dominant somatostatin receptor subtype expressed by inflammatory cells, is widely expressed and directly regulates T cell IFN-gamma release. Eur J Immunol. 1999;29:2454-2463.

14. Likar Y, Zurita J, Dobrenkov K, et al. A new pyrimidine-specific reporter gene: a mutated human deoxycytidine kinase suitable for PET during treatment with acycloguanosine-based cytotoxic drugs. J Nucl Med. 2010;51:1395-1403. 
15. Riviére I, Sadelain M. Chimeric antigen receptors: a cell and gene therapy perspective. Mol Ther. 2017;25:1117-1124.

16. Ellis J. Silencing and variegation of gammaretrovirus and lentivirus vectors. Hum Gene Ther. 2005;16:1241-1246.

17. Riviére I, Dunbar CE, Sadelain M. Hematopoietic stem cell engineering at a crossroads. Blood. 2012;119:1107-1116.

18. von Kalle C, Deichmann A, Schmidt M. Vector integration and tumorigenesis. Hum Gene Ther. 2014;25:475-481.

19. Urnov FD, Rebar EJ, Holmes MC, Zhang HS, Gregory PD. Genome editing with engineered zinc finger nucleases. Nat Rev Genet. 2010;11:636-646.

20. Sander JD, Joung JK. CRISPR-Cas systems for editing, regulating and targeting genomes. Nat Biotechnol. 2014;32:347-355.

21. Guilinger JP, Pattanayak V, Reyon D, et al. Broad specificity profiling of TALENs results in engineered nucleases with improved DNA-cleavage specificity. Nat Methods. 2014;11:429-435.

22. Eyquem J, Mansilla-Soto J, Giavridis T, et al. Targeting a CAR to the TRAC locus with CRISPR/Cas9 enhances tumour rejection. Nature. 2017;543:113117.
23. Rafiq S, Yeku OO, Jackson HJ, et al. Targeted delivery of a PD-1-blocking scFv by CAR-T cells enhances anti-tumor efficacy in vivo. Nat Biotechnol. 2018;36:847-856.

24. Tavaré R, Escuin-Ordinas H, Mok S, et al. An effective immuno-PET imaging method to monitor CD8-dependent responses to immunotherapy. Cancer Res. 2016;76:73-82.

25. Ronald JA, Kim BS, Gowrishankar G, et al. A PET imaging strategy to visualize activated $\mathrm{T}$ cells in acute graft-versus-host disease elicited by allogenic hematopoietic cell transplant. Cancer Res. 2017;77:2893-2902.

26. Markovic SN, Galli F, Suman VJ, et al. Non-invasive visualization of tumor infiltrating lymphocytes in patients with metastatic melanoma undergoing immune checkpoint inhibitor therapy: a pilot study. Oncotarget. 2018;9:3026830278.

27. Bensch F, van der Veen EL, Lub-de Hooge MN, et al. ${ }^{89} \mathrm{Zr}$-atezolizumab imaging as a non-invasive approach to assess clinical response to PD-L1 blockade in cancer. Nat Med. 2018;24:1852-1858.

28. Ponomarev V. Advancing immune and cell-based therapies through imaging. Mol Imaging Biol. 2017;19:379-384. 